\title{
Synthesis and characterization of mullite-type NdMnTiO5: structural, spectroscopic, thermogravimetric and magnetic susceptibility analyses
}

\author{
Kowsik Ghosh¹, M. Mangir Murshed ${ }^{1,3}$, Debabrata Nayak ${ }^{1}$, Thomas Frederichs ${ }^{2}$, Thorsten M. Gesing ${ }^{1,3}$ \\ ${ }^{1}$ University of Bremen, Institute of Inorganic Chemistry and Crystallography, Leobener Straße 7, D-28359 Bremen, Germany \\ ${ }^{2}$ University of Bremen, Faculty of Geosciences, D-28359 Bremen, Germany \\ ${ }^{3}$ MAPEX Center for Materials and Processes, Bibliothekstraße 1, Universität Bremen, D-28359 Bremen, Germany
}

ghosh@uni-bremen.de

Members of the multiferroic $R \mathrm{Mn}_{2} \mathrm{O}_{5}(R=\mathrm{Y}, \mathrm{Bi}$ and rare earth elements) family are well known for their concomitant presence of more than one order parameters at a given temperature [1]. Due to centrosymmetric structure of the mullite-type $\mathrm{BiMn}_{2} \mathrm{O}_{5}$ compound the microscopic origin of the multiferroicity was explained in terms of complex interplay between spin-ordering, highly polarizable $\mathrm{Bi}^{3+}$ with stereo-chemically active lone electron pair, $\mathrm{Mn}^{3+} / \mathrm{Mn}^{4+}$ charge-ordering and geometric distortions of the $\mathrm{MnO}_{y}$ coordination polyhedra. A cooperative antiferromagnetic (AFM) ordering between $\mathrm{M}^{3+}$ and $\mathrm{Nd}^{3+}$ was also observed for $\mathrm{NdCrTiO}_{5}[2]$ and $\mathrm{NdFeTiO}_{5}$ $[3,4]$. Below the respective $T_{N}$ the $\mathrm{M}^{3+}$ cations become AFM and turn the $\mathrm{Nd}^{3+}$ cations into AFM along the $a b$ plane through exchange coupling [2]. The collinearly ordered $\mathrm{M}^{3+}$ cations along the octahedral chain directing $\mathbf{c}$-axis gives rise to magnetostriction, leading to multiferroicity in this compound [2]. In search of novel multiferroics, we report the synthesis and characterization of the mullite-type O10 phase isostructural $\mathrm{NdMnTiO}_{5}$ compound. The crystal structural features are described using X-ray powder diffraction data Rietveld refinements (Fig. 1). Complementary optical phonon analyses were carried out by infrared and Raman spectroscopy. The optical bandgap was obtained using both Tauc and recently introduced DASF [5-6] methods to determine type and energy of the transition, respectively. The enhancement of DC magnetic susceptibility is a common feature in rare-earth manganates. The Fisher's heat capacity shows clear evidence of the onset of long range ordering. The large deviation between ZFC and FC susceptibility below the Néel temperature $(43(1) \mathrm{K})$ indicates the presence of competing interactions and/or magnetic anisotropy in the orthorhombic system. Assuming weak interactions between $\mathrm{Nd}^{3+}$ and $\mathrm{Mn}^{3+}$ magnetic sublattices, the effective total magnetic moment was calculated from the temperature-dependent paramagnetic region, which lies close to theoretical spin-only magnetic moment values. The temperaturedependent magnetic susceptibility was modelled using the mean field approximation, where an interaction between the ordered $\mathrm{Mn}^{3+}$ spins and the electrons occupying the lowest lying Kramers' doublet of the $\mathrm{Nd}^{3+}$ cations was considered.
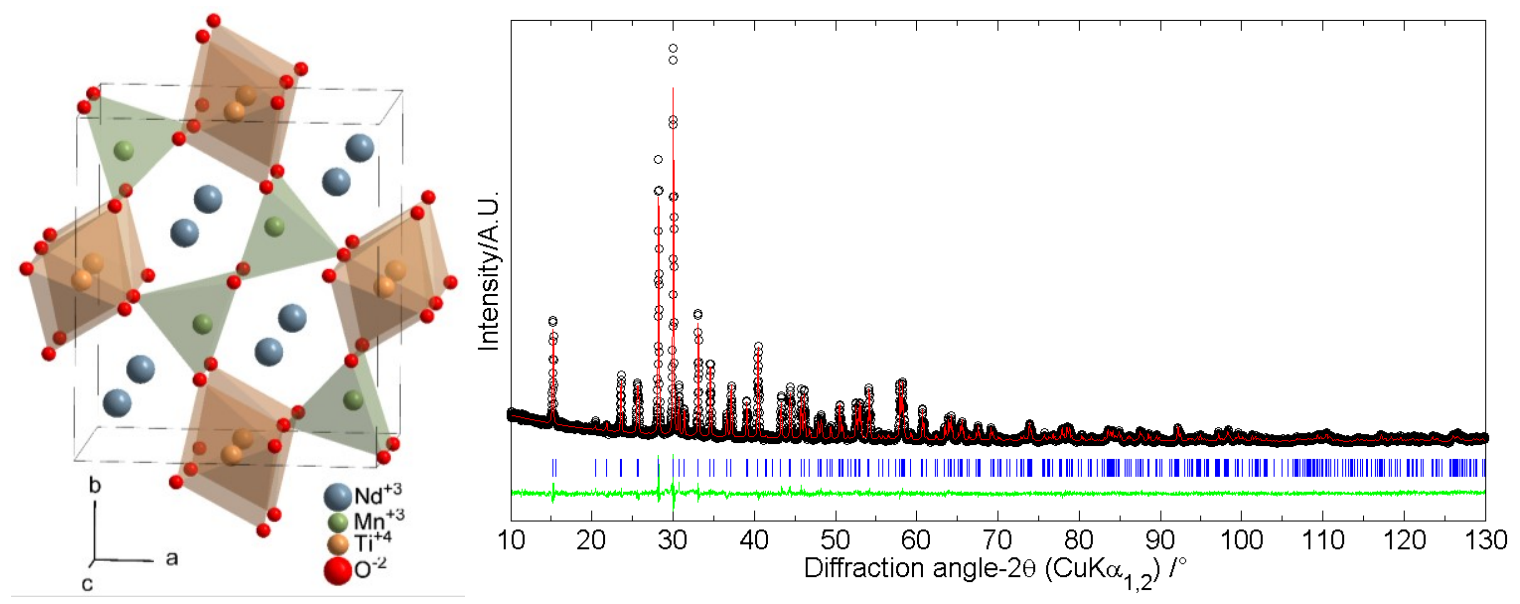

Figure 1: Crystal structure (left) and X-ray powder data Rietveld refinement of $\mathrm{NdMnTiO}_{5}$ (right).

[1] A. Munoz, J.A. Alonso, M.T. Casais, M.J. Martinez-Lope, J.L. Martinez, M.T. Fernandez-Diaz (2002). Phys. Rev. B 65 , 144423.

[2] J. Saha, G. Sharma, P. Patnaik (2014). J. Magn. Magn. Mater. 360,34.

[3] G. Buisson (1970). J. Phys. Chem. Solids 31, 1171.

[4] I. Yaeger (1978). J. Appl. Phys. 49, 1513.

[5] A. Kirsch, MM: Murshed, M.J. Kirkhame, A. Huq, J.F. Litterst, Th.M. Gesing (2018). J Phys. Chem. C. $122,28280$.

[6] A. Kirsch, MM: Murshed, J.F. Litterst, Th.M. Gesing (2019). J Phys. Chem. C. 123, 3161.

Keywords: multiferroic; crystal structure; optical phonon; bandgap; magnetic property. 